\title{
ORIGINAL ARTICLE Sexy males and sexless females: the origin of triploid apomicts
}

\author{
P Muralidhar and D Haig
}

Apomixis and polyploidy are closely associated in angiosperms, but the evolutionary reason for this association is unknown. Taraxacum officinale, the common dandelion, exists both as diploid sexuals and triploid apomicts. Here, in the context of $T$. officinale, we provide a model of the evolution of triploid apomicts from diploid sexuals. We posit an apomictic allele that arrests female meiosis in diploids, so that the plant produces diploid egg cells that can develop without fertilization, but haploid pollen. We propose occasional fertilization of diploid egg cells by haploid pollen, resulting in triploid apomicts that produce triploid egg cells but largely nonfunctional pollen. The irreversibility of this process renders diploid partial apomicts evolutionarily short-lived, and results in fixation of triploid apomicts except when they suffer extreme selective disadvantages. Our model can account for the high genetic diversity found in $T$. officinale triploid populations, because recombinant haploid pollen produced by diploids allows the apomictic allele to spread onto many genetic backgrounds. This leads to multiple clonal lineages in the newly apomictic population, and thereby alleviates some of the usual pitfalls of asexual reproduction.

Heredity (2017) 118, 436-441; doi:10.1038/hdy.2016.124; published online 11 January 2017

\section{INTRODUCTION}

Asexual reproduction via seeds (apomixis) has evolved many times in flowering plants (Richards, 2003; van Dijk, 2003; Whitton et al., 2008; Hörandl and Hojsgaard, 2012) and is commonly associated with increased ploidy (Bierzychudek, 1985; Whitton et al., 2008). Obligately asexual species are often claimed to enjoy an initial advantage over their sexual relatives owing to greater reproductive efficiency (the 'twofold cost of sex') and assurance (no need for mate finding), followed by a long-term evolutionary disadvantage from lack of adaptability (Darlington, 1939; Maynard Smith, 1978). However, in hermaphroditic plants, the twofold increase in genetic representation in apomictic seeds, relative to sexual seeds, would seem to be balanced by the loss of reproduction via male (pollen) function, for no net advantage (Mogie, 1996). The initial advantage of apomixis seems more likely to be the preservation of high-quality genotypes by the suppression of sexual recombination. Consistent with this, apomictic lineages occupy more extensive geographic ranges than their sexual relatives, especially at higher latitudes (Bierzychudek, 1985; van Dijk, 2003). Consistent with a long-term disadvantage, apomictic plants tend to occupy the 'twigs' of phylogenetic trees, nested within sexual clades (van Dijk, 2003).

The geographical supremacy of apomictic lineages, given that they are usually polyploid, could also be explained by advantages of polyploidy itself: it can confer a physiological advantage in harsh environments (Levin, 1983; te Beest et al., 2012), increase the rate of a population's adaptation (Otto, 2007) and act as a buffer against deleterious mutations, at least in the short term (Mogie and Ford, 1988; King et al., 2012; Soltis et al., 2014; Selmecki et al., 2015; Saccucci et al., 2016). However, aside from any general advantages and disadvantages of sex and polyploidy, the ability to reproduce asexually is clearly advantageous to polyploid individuals that are unable to produce viable haploid spores by conventional meiosis. However, there is no obvious reason why polyploidy should directly induce apomixis, and hence the repeated association of the two phenomena remains a mystery.

Taraxacum officinale, the common dandelion, is a model system for studying the evolution of apomixis (Richards, 2003). Sexual dandelions are hermaphroditic, with individual plants producing both pollen and egg cells (Grimanelli et al., 2001; van Dijk, 2003). In Europe, T. officinale exists as both diploid sexual populations and triploid apomictic clones that produce little, if any, functional pollen (Mogie and Ford, 1988; van Dijk, 2003; van Dijk and Bakx-Schotman, 2004). In contrast, all T. officinale in North America are apomictic triploids (Lyman and Ellstrand, 1998). Triploid T. officinale are autonomous gametophytic apomicts, meaning that embryo sacs develop from unreduced megaspores and both endosperm and embryo are produced without fertilization (Grimanelli et al., 2001). T. officinale in Europe and Asia exhibit the common pattern of geographical parthenogenesis in which apomicts are more widely distributed than sexual forms (Mogie and Ford, 1988; van Dijk, 2003). The wellstudied natural history of this species, along with the stable existence of both apomictic triploid and sexual diploid populations, make it an ideal taxon on which to base a model of the concomitant evolution of apomixis and polyploidy.

Populations of apomictic dandelions are clonally diverse, contrary to the intuitive expectation that asexual populations should be genetically homogenous (Van der Hulst et al., 2003; van Dijk, 2003; Majeský et al., 2012). Although mutational divergence will eventually 
result in clonal diversity, this process is likely to occur over long periods of evolutionary time. High clonal diversity of triploid apomicts could result from the rare recruitment of new apomictic clones through the occasional fertilization of haploid egg cells in sexual diploids with diploid pollen from triploid apomicts. Alternatively, this diversity could arise from many origins of apomicts from an original sexual population with multiple lineages maintained over evolutionary time.

Here, we propose a model of the origin and spread of apomixis in T. officinale in which apomictic seed production originates in diploid individuals that reproduce sexually via pollen, thus spreading the apomictic trait onto diverse genetic backgrounds. Occasional fertilization of apomictic diploid egg cells by haploid pollen of any genotype generates new triploid apomicts that no longer produce functional pollen. We explore the model's dynamics under a variety of selection regimes. We find that one equilibrium state of the system is a diverse population of triploid apomicts without diploid individuals. This provides an explanation of clonal diversity among apomicts that does not require rare function of pollen from triploid apomicts (Tas and van Dijk, 1999). The equilibrium states of our model are unchanged if multiple loci form the genetic basis of apomixis, although the rate of the spread of apomixis is slowed because of recombination and segregation in pollen production. This is because the entire genome functions as a single locus in apomictic reproduction. Although our model is based on the biology of $T$. officinale, it can provide insight into transitions in ploidy and sexuality across the tree of life (Beaton and Hebert, 1988; Neiman et al., 2011).

For simplicity, our model assumes that triploid apomicts produce no viable pollen. Most pollen produced by $T$. officinale triploid apomicts is aneuploid and inviable, but experimental crosses between diploid sexual dandelions and triploid apomictic dandelions show that triploid apomicts produce a small proportion of viable euploid pollen (haploid, diploid or triploid; Tas and van Dijk, 1999). This rare euploid pollen could serve as a vector for the transmission of apomixis into a diploid sexual population in the presence of triploid apomicts. We would expect to observe relatively more functional diploid than haploid pollen in lineages that have been apomictic for many generations because of the gradual accumulation of deleterious recessive mutations that would be exposed and selected against in haploid pollen, but masked in diploid pollen (Tas and van Dijk, 1999; van Dijk, 2003).

Our model is the first theoretical explanation of the dual origin of apomixis and triploidy in T. officinale, and can also explain the high clonal diversity in newly apomictic populations. Previous models have suggested that triploid diversity in this species is generated by the fertilization of haploid egg cells of sexual females by diploid pollen produced by previously apomictic males (van Dijk, 2003). In the model we propose here, triploid diversity is initially generated by the fertilization of diploid egg cells of apomictic females by haploid pollen of sexual males. However, these two models of gene flow-the hybridization of apomictic populations with sexual populations or the origin and spread of an apomictic allele within a population-are reciprocal. Both processes may have contributed to the current observed diversity of established populations of triploids in T. officinale.

\section{MATERIALS AND METHODS}

We assume a single dominant locus that allows the production of apomictic seeds, akin to the Diplosporous genomic region identified in $T$. officinale (Bicknell et al., 2000; Richards, 2003; van Dijk and Bakx-Schotman, 2004; Vijverberg et al., 2010). However, this locus may also represent a set of tightly linked genes associated in function, such as a gene controlling apomeiosis (meiotic arrest resulting in unreduced gametes) linked with a gene controlling parthenogenesis (development of an unfertilized egg cell; van Dijk and BakxSchotman, 2004). This locus has two alleles, an original recessive $a$ and a dominant allele $A$ causing the production of diploid egg cells with identical Aa genotype of the parent plant. This allele may function on a genomic background that includes other alleles that enable apomixis, rather than being solely responsible for the trait (Whitton et al., 2008). The production of diploid egg cells would likely occur by some form of arrest in female meiosis, but does not apply to pollen production (van Dijk and Bakx-Schotman, 2004). Individuals with $A a$ genotypes will therefore produce egg cells with $A a$ genotypes, but normal haploid pollen with an $A$ or a genotype in equal proportions (diploid $a a$ individuals produce both haploid $a$ egg cells and haploid $a$ pollen).

We allow the $A a$ egg cells of the diploid apomicts to be fertilized at some frequency $u$ that then results in triploid $A a a$ or $A A a$ individuals, depending on the genotype of the haploid pollen (Tas and van Dijk, 1999). This parameter can vary according to the difficulty of fertilizing an unreduced egg cell. Triploid individuals can only reproduce apomictically, and therefore only produce triploid seeds of identical genotypes and almost no functional pollen; we do not consider the production of rare euploid pollen by triploids in our model (Tas and van Dijk, 1999; van Dijk and Bakx-Schotman, 2004). Rare tetraploid individuals are sometimes detected in natural populations (Verduijn et al. 2004) but, for simplicity, we do not consider the fertilization of triploid (or higher ploid) apomicts or the fertilization of haploid egg cells by triploid pollen. Our model also includes viability selection against both triploid apomicts, $A a a$ and $A A a$, and diploid apomicts (denoted by the variables $t, f$ and $s$, respectively), reflecting a situation in which reduced genetic variation among an individual's offspring may lead to increased susceptibility to pathogens or predators (Kovel and Jong, 2000; Table 1). We assume random mixing of pollen and egg cells in order to determine frequencies in the next generation.

If $P$ and $Q$ are the respective population frequencies of sexual and apomictic diploids in a given generation, and $s$ is the viability disadvantage of the diploid apomicts relative to the sexual diploids, then the proportions of $A$ and $a$ pollen in the next generation are:

$$
\begin{aligned}
& p_{A}=(1-s)(Q / 2) /[P+(1-s) Q] \\
& p_{a}=1-P_{A}=[P+(1-s)(Q / 2)] /[P+(1-s) Q](\text { Table } 1)
\end{aligned}
$$

If, further, $R$ and $K$ are the respective frequencies of $A a a$ and $A A a$ triploid apomicts (so that $P+Q+R+K=1$ ), whose viability disadvantages relative to sexual diploids are $t$ and $f$, respectively, then seeds of genotypes $a, A a, A a a$ and $A A a$ are produced in proportions:

$$
\begin{aligned}
& q_{a}=P /[P+(1-s) Q+(1-t) R+(1-f) K] \\
& q_{A a}=(1-s) Q /[P+(1-s) Q+(1-t) R+(1-f) K] \\
& q_{A a a}=(1-t) R /[P+(1-s) Q+(1-t) R+(1-f) K] \\
& q_{A A a}=(1-f) K /[P+(1-s) Q+(1-t) R+(1-f) K]
\end{aligned}
$$

A proportion $u$ of the diploid egg cells is fertilized by pollen, and hence the population frequencies of plants of the various genotypes in the next generation are:

$$
\begin{aligned}
& P^{\prime}=p_{a} q_{a} \\
& Q^{\prime}=p_{A} q_{a}+(1-u) q_{A a} \\
& R^{\prime}=q_{A a a}+u p_{a} q_{A a} \\
& K^{\prime}=q_{A A a}+u p_{A} q_{A a}
\end{aligned}
$$

We ran our model under several different selective regimes in order to determine the evolutionary dynamics of the apomictic allele, $A$, when introduced at low frequency $(0.01 \%)$ into a population of diploid sexuals. These selective regimes included selection against apomicts ( $A a, A a a$ and $A A a$ individuals; $s=t=f>0$ ); selection because of the apomictic allele 
( $A a, A a a$ and $A A a$ individuals; $s=t=f / 2>0$ ); selection against triploids ( $A a a$ and $A A a$ individuals; $s=0, t=f>0$ ); and selection against diploid apomicts ( $A a$ individuals; $s>0, t=f=0$ ). Under each scenario, we assigned different values to the selective coefficients against each genotype class (Table 1), and observed the equilibrium frequency of the genotypes in the population. All simulations described in this paper were run in Matlab (version R2016a, MATLAB and Statistics Toolbox Release, 2016a).

\section{RESULTS}

\section{Selection against apomicts}

We begin with a case in which equal selective disadvantages were assigned to all of the apomicts $(s=t=f>0)$. For selection coefficients from 0 to $1 / 3$ (where selection is expressed as $1-s$ ), the triploid apomicts take over the population (Figure 1). This transition occurred rapidly, with partially apomictic diploids present for only a transient phrase in the population, rising in frequency rapidly as the $A$ allele is introduced into the population and then steadily declining (Figure 1). When $s>1 / 3$, diploid apomicts do not become established in the population and sexual diploids remain the only genotype. Overall, high selective disadvantages are needed to prevent the rise in frequency of diploid partial apomicts and the subsequent rise in frequency of triploid apomicts. Although the rate of fertilization of diploid apomicts, $u$, determines the length of time in which they exist in the population, it does not qualitatively change our results; triploid apomicts still take over the population.

Logically, if diploid and triploid apomicts share the same selective disadvantage, the diploid apomicts will be converted to triploid apomicts at a constant frequency $u$, which is the fertilization rate of diploid egg cells in our model. As the triploid apomicts are an absorbing state in this model, they will inevitably rise in frequency holding all other factors constant. Any selective advantage in favor of the apomicts will only enhance this path to fixation. Separating the triploid apomicts into two categories ( $A a a$ and $A A a$ ) reveals that triploids with two recessive alleles will rise in frequency before triploids with two dominant alleles, because of the initially high frequency of $a$ in the pollen population. As diploid apomicts replace diploid sexuals in the population of haploid pollen producers, however, new $A a a$ and $A A a$ triploids will be generated in equal frequencies.

\section{Selective disadvantage because of the apomictic allele}

In the next scenario, we ran the model assuming that the $A$ apomictic allele carries an additive selective disadvantage, possibly because of pleiotropic effects in other aspects of reproduction (Joshi and Moody, 1998). In this scenario, $A A a$ has twice the selective disadvantage of $A a$ and $A a a(s=t=f / 2>0)$. As expected, Aaa eventually reaches fixation after the transitory appearance of $A A a$ individuals (Figure 2).

Table 1 The genotypes and associated phenotypes used in our model of apomixis, along with the variables representing each genotype in our Equations

\begin{tabular}{llll}
\hline Genotype & Phenotype & Frequency & Viability \\
\hline aa & Diploid sexual & $P$ & 1 \\
Aa & Diploid partial apomict & $Q$ & $1-s$ \\
Aaa & Triploid apomict & $R$ & $1-t$ \\
$A A a$ & Triploid apomict & $K$ & $1-f$ \\
\hline
\end{tabular}

The selection coefficients used in our model are listed under viability and are expressed as the selective disadvantage of each genotype from a maximum fitness of 1 (see Materials and Methods for more detail).
Extending this result to nonadditive cases, $A A a$ will be lost in any case where its selective disadvantage is greater than that of the Aaa triploid. This scenario is not qualitatively different for the diploid apomict that follows a pattern of briefly reaching high frequencies, but then gradually disappearing. This pattern of a single triploid genotype resembles empirical observations of the gene Diplosporous (van Dijk and Bakx-Schotman, 2004). The Diplosporous allele occurs in single dose in triploid apomictic dandelions (van Dijk and Bakx-Schotman, 2004). Triploids have the genotype $D d d$ where $D$ is a dominant allele controlling the production of unreduced megaspores and thus disrupting traditional meiosis (van Dijk and Bakx-Schotman, 2004).
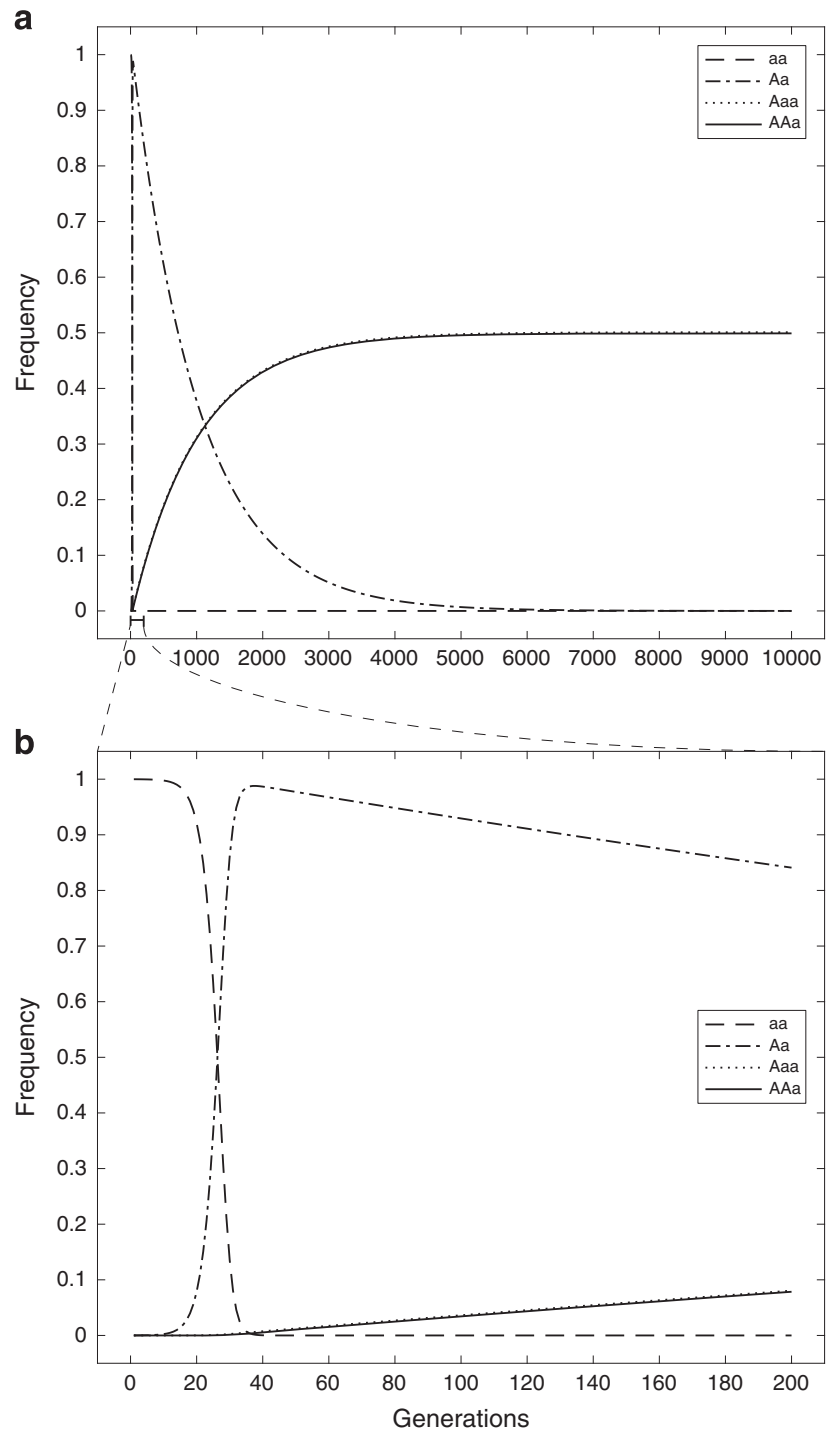

Figure 1 The gradual takeover by triploid apomicts in a population of sexual diploids, given selection against all apomictic types. The Aa genotype was introduced into the population at an initial frequency of 0.0001 , with a proportion $u=0.001$ of diploid egg cells fertilized each generation. The selective coefficients against the three apomictic genotypes were equal at 0.05. (a) The dynamics of this selection regime across 10000 generations that reach an equilibrium state with only the triploid genotypes. (b) Focusing on the first 200 generations allows it to be more clearly seen that diploid sexuals are rapidly replaced by diploid partial apomicts, that are in turn converted into triploid apomicts. 
Furthermore, no genotypes of the form $A A a$ have been found in any previously studied apomictic plant (Ozias-Akins and van Dijk, 2007).

\section{Selection against triploids}

We then examined a scenario in which triploidy carried a selective disadvantage, whereas $A a$ diploid apomicts suffered no selective disadvantage $(s=0, t=f>0)$. This resulted in the stable coexistence of $A a, A a a$ and $A A a$ apomicts. The relative frequencies of the three genotypes at selective equilibrium depended on $s, t$ and $f$. Figure 3 illustrates an example with realistic selective coefficients where the three genotypes approach similar frequencies. With very high selection against triploids, diploid apomicts predominate.

This scenario can be thought of in terms of a heterozygote advantage; the diploid apomicts are constantly transforming into triploids at the fertilization rate $u$, but their relative selective advantage prevents them from vanishing as they did in the previous scenario. Diploid apomictic dandelions have not been reported in natural populations. In terms of our model, this suggests that triploid apomicts cannot have experienced a major selective disadvantage relative to diploids.

\section{Selection against diploid apomicts}

Finally, we consider the scenario in which diploid apomicts have a selective disadvantage relative to both the sexual diploids and the triploid apomicts $(s>0, t=f=0)$. This scenario seems the least biologically relevant, but might occur if there is an intrinsic conflict between apomictic and sexual reproduction in the same plant that results in an overall fitness loss for the individual. If the previous selection against triploids resembled a heterozygote advantage case, this scenario resembles a heterozygote disadvantage situation. In this case, the population eventually consists of triploids only as long as selection against diploid apomicts is less than $1 / 3$. However, if there is no selective disadvantage against either triploid, the population will have a higher frequency of the $A a a$ genotype than the $A A a$ genotype (Figure 4). This is explained by the relative lack of $A$ pollen in the

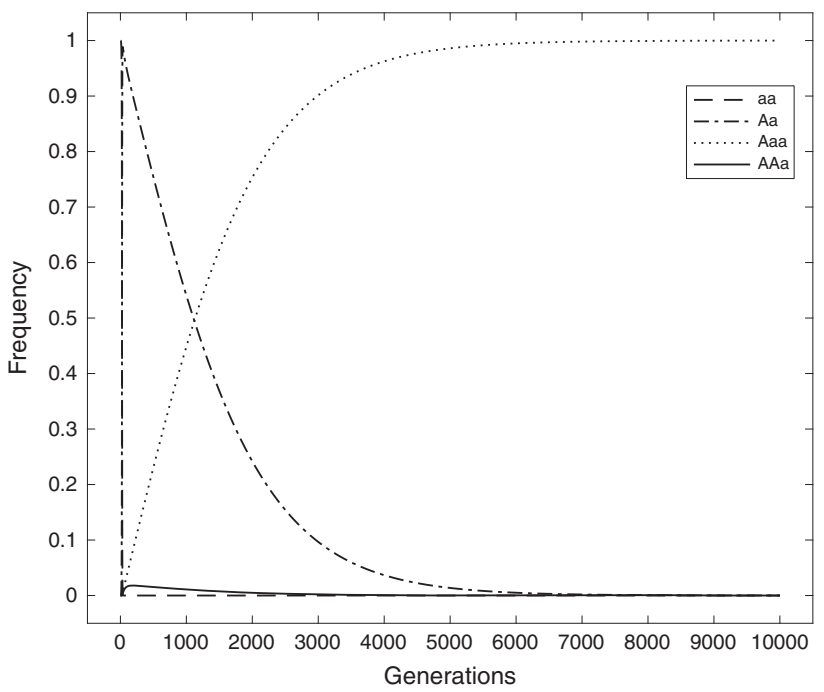

Figure 2 Fixation of Aaa triploid apomicts in a population of sexual diploids, given additive selection against the $A$ allele. The $A a$ genotype was introduced into the population at an initial frequency of 0.0001 , with a proportion $u=0.001$ of diploid egg cells fertilized each generation. The selective coefficients against the $A a$ and Aaa genotypes were 0.025 , whereas that against the $A A$ a genotype was 0.05 . population because of the selective disadvantage of the diploids, resulting in the fixation of the more common Aaa genotype (that only depends on a pollen). Again, this pattern mimics empirical evidence from the Diplosporous gene, suggesting another possibility for the fixation of a single triploid Ddd genotype at that locus (van Dijk and Bakx-Schotman, 2004). As selection against triploids increases, this scenario approaches general selection against apomicts, and both triploid genotypes reach equal frequencies in the population.

\section{Relaxing the conditions for success of apomixis by adding complexity}

Our model assumes fixed selection coefficients for the apomictic genotypes relative to sexual diploids. However, in a more realistic model, the average fitness of the apomictic population as a whole would increase over time with the generation of new apomictic lineages from the sexual population. The fitness of an allele in a sexual population is averaged across genetic backgrounds because multilocus genotypes are being continually recombined. Thus, alleles in sexual populations change in frequency according to their average effect on fitness (narrow-sense heritability of fitness). In contrast, each apomictic lineage perpetuates itself indefinitely and changes in frequency according to the fitness of its unique multilocus genotype (broad-sense heritability of fitness). Given the sexual spread of the gene for apomixis onto new genetic backgrounds via pollen, the population of apomicts will come to be dominated by elite non-recombining genotypes.

Apomictic reproduction in dandelions requires the formation of unreduced egg cells (apomeiosis) and the development of embryos from these egg cells without fertilization (parthenogenesis). Our model treats both these traits as residing in a single allele $A$ at a single locus. The absence of recombination in apomictic reproduction relaxes the requirement for a supergene that determines both apomictic characters. Assume that diploid apomixis occurs in $A a B b$ genotypes, where A represents an allele causing female apomeiosis and B represents an allele allowing parthenogenesis. Given the origin of the first such

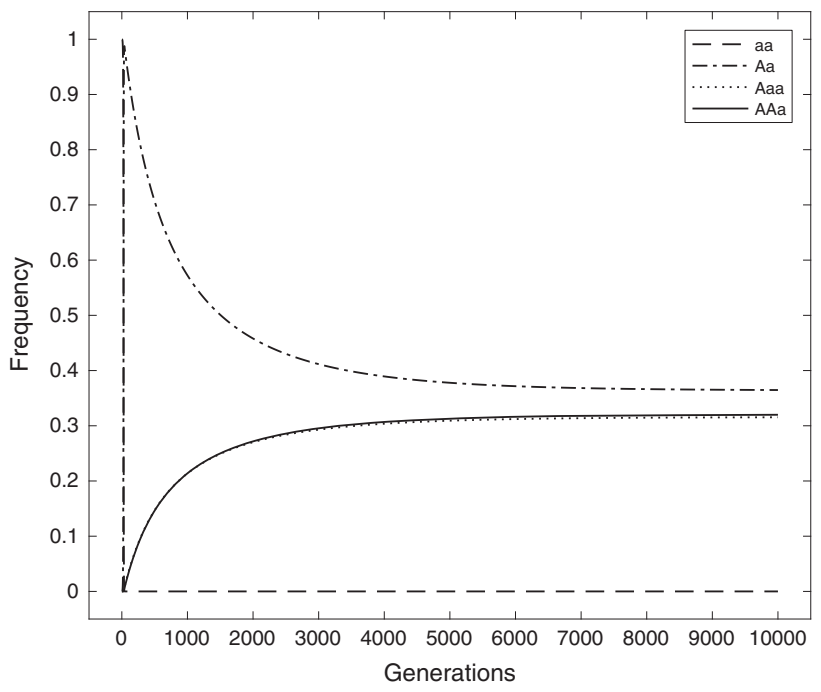

Figure 3 Stable coexistence of all three apomictic genotypes in the population, given a selective disadvantage for triploidy. The Aa genotype was introduced into the population at an initial frequency of 0.0001 , with a proportion $u=0.001$ of diploid egg cells fertilized each generation. The selective coefficients against the $A A a$ and Aaa genotypes were 0.05, and that against the Aa genotype was 0.0485 . 


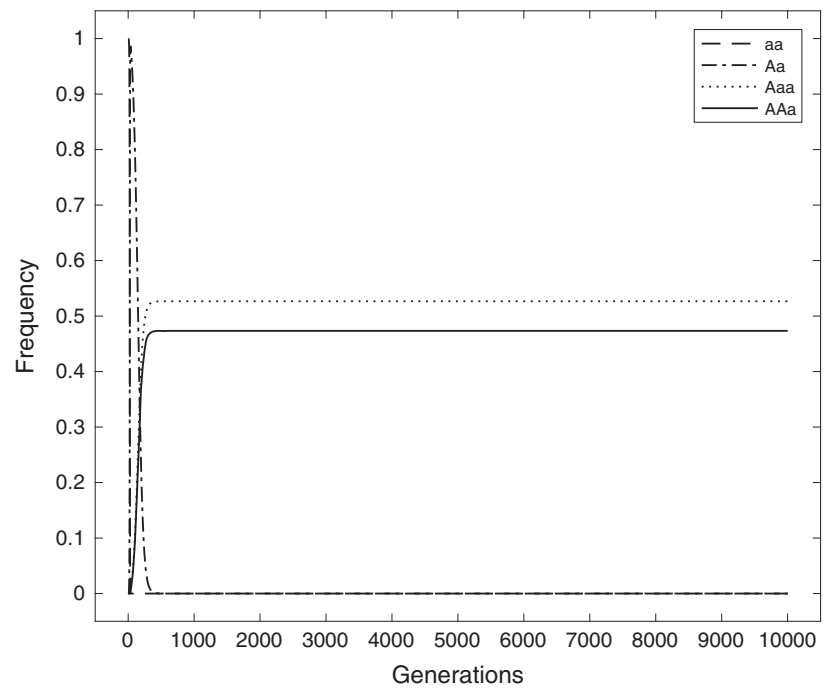

Figure 4 Different frequencies of the Aaa and $A A a$ triploid resulting from selection against only the diploid apomict. The Aa genotype was introduced into the population at an initial frequency of 0.0001 , with a proportion $u=0.001$ of diploid egg cells fertilized each generation. The selective coefficients for the $A A a$ and Aaa genotypes were 0 , and the selective coefficient against the Aa genotype was 0.025.

genotype, perhaps by the chance co-occurrence of two separate mutations in the same individual, apomixis would spread through the population by fertilization of diploid sexuals by $A B$ pollen. If the two loci were unlinked, this would compose a quarter of the pollen produced by $A a B b$ diploid apomicts. The rate of spread of apomixis into the sexual population would be slowed, relative to the single-locus model, but the basic dynamics of the spread of apomixis and the conversion of diploid apomicts to male-sterile triploid apomicts would be unchanged.

Our model also assumes that triploid apomicts produce no viable pollen. Most pollen produced by triploid apomicts is aneuploid and inviable, but experimental crosses between diploid sexual dandelions and triploid apomictic dandelions show that triploid apomicts produce a small proportion of viable euploid pollen (Tas and van Dijk, 1999). This rare euploid pollen could serve as a transmission mechanism of apomixis into a diploid sexual population in the presence of triploid apomicts. We would expect to observe a greater frequency of functional diploid, rather than haploid, pollen in lineages that have been apomictic for many generations because of the gradual accumulation of deleterious recessive mutations that would be exposed and selected against in haploid pollen, but masked in diploid pollen (Tas and van Dijk, 1999; van Dijk, 2003).

\section{DISCUSSION}

In the scenarios we examined, triploid apomicts invade a mixed population of sexual and apomictic diploids in almost every case, barring a strong selective disadvantage of triploidy. Surprisingly, diploid apomicts that are able to reproduce both sexually and asexually are usually unable to persist in the population unless they have substantially higher survival than triploids that cannot reproduce sexually. The ability of diploid apomicts to reproduce by both means is cancelled out by their irreversible conversion to triploids by occasional fertilization of diploid egg cells by haploid pollen. In our model, this conversion occurs at the constant rate $u$. In reality, the rate of fertilization of diploid egg cells would decline as the proportion of diploid pollen producers declined. This may indicate a selective advantage to sexual diploids in natural populations of $T$. officinale, enabling them to overcome a constant conversion into triploid apomicts, or a reciprocal return to sexual reproduction in apomictic populations (Sørensen, 1958).

Our model provides a path for the evolution of triploid apomicts that does not require a causal role for triploidy in apomixis. Given the rapid conversion of diploid apomicts to triploids determined by the rate of diploid fertilization, we also do not expect prolonged persistence of diploid apomicts within a population. Further investigation into the structure of mixed T. officinale populations in Central Europe may provide evidence for this transitional state (van Dijk et al., 2009). Though our model has two possible triploid genotypes ( $A A a$ and $A a a$ ), any selective differences between them would result in one eventually outcompeting the other. A cost to the extra apomictic allele in $A A a$ apomicts, and therefore the relative dominance of the Aaa genotype, is consistent with the observation that triploid apomictic dandelions carry only one copy of the Diplosporous allele (van Dijk and Bakx-Schotman, 2004). The existence, although rare, of viable tetraploids lends support to our model, suggesting that occasional fertilization of triploid egg cells by haploid pollen, or of haploid egg cells by triploid pollen, may be possible.

Previous explanations of clonal diversity among apomictic Taraxacum have focused on the fertilization of haploid sexual egg cell by diploid pollen derived from apomicts, providing a mechanism for gene flow from sexual diploids into the population of apomicts (van Dijk, 2003). However, this process does not explain the origin of apomictic triploids. Our model suggests that apomictic triploids could initially have been produced by the fertilization of unreduced diploid egg cells by haploid sperm. Because our model describes the initial evolution of apomixis, recessive deleterious mutations would not have had time to accumulate and haploid pollen containing the apomictic allele would still be viable. Although the combination of pollen production by a triploid individual and the transmission of this pollen to a new population is likely infrequent, there is some evidence to suggest that apomictic polyploid plants may occasionally act as viable pollen donors and enable the spread of apomixis across populations (Morita et al., 1990; Menken et al., 1995; van Dijk et al., 2009).

Instead of the spread of a single apomictic allele through multiple populations, the representative locus in our model could be a gene with recurrent mutations that result in apomictic seed production. This could occur in a gene controlling meiosis in the embryo sac, in which a disrupting mutation against the correct genetic background could result in an unreduced apomictic egg cell. This apomeiotic allele must occur against a parthenogenetic background to result in an apomictic phenotype. This scenario is partially supported by experiments to isolate the locus controlling apomixis in Taraxacum; the Diplosporus locus appears to be large, and mutations in this region may be associated with meiotic arrest and provide the initial step in the path to apomictic reproduction (van Dijk and Bakx-Schotman, 2004; Vijverberg et al., 2010; Vašut et al., 2014).

In either case, our model provides an explanation for the clonal diversity found among apomictic lines, as they could originate from multiple diploid apomicts with different recessive haplotypes. This overcomes some of the evolutionary cost of Darlington's 'blind alley' of apomixis, making these species partially robust to environmental perturbations selecting for phenotypic diversity and suggesting a novel explanation for their wide distribution across multiple continents (Darlington, 1939; van Dijk, 2003).

We recognize the oddity of invoking a phenotype (diploid malefertile apomicts) that has not been observed in natural populations of 
dandelions to explain the origin of triploid apomicts, although diploid apomicts have been observed in other taxa such as Hieracium (Bicknell, 1997) and Boechera (Lovell et al., 2013). However, our model also explains why we should fail to observe this phenotype, as their presence in a population is predicted to be ephemeral. If diploid male-fertile apomicts were to arise within a population, then their initial success is predicted to be terminated by their replacement by their own triploid apomictic progeny. In addition, although we have focused on the transition to sexual diploids to apomictic triploids in T. officinale, our model can be applied to a range of organisms with similar transitions from sexual to asexual reproduction associated with higher ploidy levels (Beaton and Hebert, 1988; Neiman et al., 2011).

\section{CONFLICT OF INTEREST}

The authors declare no conflict of interest.

\section{ACKNOWLEDGEMENTS}

We thank Carl Veller and Peter Wilton for helpful comments.

Beaton MJ, Hebert PD (1988). Geographical parthenogenesis and polyploidy in Daphnia pulex. Am Nat 132: 837-845.

Bicknell RA (1997). Isolation of a diploid, apomictic plant of Hieracium aurantiacum. Sex Plant Reprod 10: 168-172.

Bicknell RA, Borst NK, Koltunow AM (2000). Monogenic inheritance of apomixis in two Hieracium species with distinct developmental mechanisms. Heredity 84: 228-237.

Bierzychudek P (1985). Patterns in plant parthenogenesis. Experientia 41: 1255-1264.

Darlington CD (1939). The Evolution of Genetic Systems. Cambridge University Press Cambridge.

Grimanelli D, Leblanc O, Perotti E, Grossniklaus U (2001). Developmental genetics of gametophytic apomixis. Trends Genet 17: 597-604.

Hörandl E, Hojsgaard D (2012). The evolution of apomixis in angiosperms: a reappraisal. Plant Biosyst 146: 681-693.

Joshi A, Moody ME (1998). The cost of sex revisited: effects of male gamete output of hermaphrodites that are asexual in their female capacity. J Theor Biol 195: 533-542.

King KC, Seppälä O, Neiman M (2012). Is more better? Polyploidy and parasite resistance. Biol Lett 8: 598-600.

Kovel D, Jong D (2000). Selection on apomictic lineages of Taraxacum at establishment in a mixed sexual-apomictic population. J Evol Biol 13: 561-568.

Levin DA (1983). Polyploidy and novelty in flowering plants. Am Nat 122: 1-25.

Lovell JT, Aliyu OM, Mau M, Schranz ME, Koch M, Kiefer C et al. (2013). On the origin and evolution of apomixis in Boechera. Plant Reprod 26: 309-315.

Lyman JC, Ellstrand NC (1998). Relative contribution of breeding system and endemism to genotypic diversity: the outcrossing endemic Taraxacum californicum vs. the widespread apomict T. officinale (sensu lato). Madrono 45: 283-289.

MATLAB and Statistics Toolbox Release (2016a). The MathWorks, Inc.: Natick, MA, USA

Maynard Smith J (1978). The Evolution of Sex. Cambridge University Press: Cambridge.
Majeský L', Vašut RJ, Kitner M, Trávníček B (2012). The pattern of genetic variability in apomictic clones of Taraxacum officinale indicates the alternation of asexual and sexual histories of apomicts. PLoS One 7: e41868.

Menken SB, Smit E, Den Nijs HCM (1995). Genetical population structure in plants: gene flow between diploid sexual and triploid asexual dandelions (Taraxacum section Ruderalia). Evolution 49: 1108-1118.

Mogie M (1996). Is there a cost of sex in hermaphrodites? Naturwissenschaften 83: 225-226.

Mogie M, Ford H (1988). Sexual and asexual Taraxacum species. Biol J Linnean Soc 35: 155-168.

Morita T, Sterk AA, Nijs J (1990). The significance of agamospermous triploid pollen donors in the sexual relationships between diploids and triploids in Taraxacum (Compositae). Plant Species Biol 5: 167-176.

Neiman M, Paczesniak D, Soper DM, Baldwin AT, Hehman G (2011). Wide variation in ploidy level and genome size in a New Zealand freshwater snail with coexisting sexual and asexual lineages. Evolution 65: 3202-3216.

Otto SP (2007). The evolutionary consequences of polyploidy. Cell 131: 452-462.

Ozias-Akins P, van Dijk PJ (2007). Mendelian genetics of apomixis in plants. Annu Rev Genet 41: 509-537.

Richards A (2003). Apomixis in flowering plants: an overview. Philos Trans $R$ Soc Lond B Biol Sci 358: 1085-1093.

Saccucci MJ, Denton RD, Holding ML, Gibbs HL (2016). Polyploid unisexual salamanders have higher tissue regeneration rates than diploid sexual relatives. J Zool 300: 77-81.

Selmecki AM, Maruvka YE, Richmond PA, Guillet M, Shoresh N, Sorenson AL et al. (2015). Polyploidy can drive rapid adaptation in yeast. Nature 519: 349-352.

Soltis DE, Visger CJ, Soltis PS (2014). The polyploidy revolution then... and now: Stebbins revisited. Am J Bot 101: 1057-1078.

Sørensen T (1958). Sexual chromosome-aberrants in triploid apomictic Taraxaca. Bot Tidskr 54: 1-22.

Tas ICQ, van Dijk PJ (1999). Crosses between sexual and apomictic dandelions (Taraxacum). I. The inheritance of apomixis. Heredity 83: 707-714.

te Beest M, Johannes Le Roux J, Richardson DM, Brysting AK, Suda J, Kubešová M et al. (2012). The more the better? The role of polyploidy in facilitating plant invasions. Ann Bot 109: 19-45.

Van der Hulst RGM, Mes THM, Falque M, Stam P, den Nijs JCM, Bachmann K (2003). Genetic structure of a population sample of apomictic dandelions. Heredity 90: 326-335.

van Dijk PJ, de Jong H, Vijverberg K, Biere A (2009). An apomixis-gene's view on dandelions. In: Schön I, Martens K, van Dijk PJ (eds). Lost Sex. Springer: Netherlands: pp 475-493.

van Dijk PJ (2003). Ecological and evolutionary opportunities of apomixis: insights from Taraxacum and Chondrilla. Philos Trans R Soc B Biol Sci 358: 1113-1121.

van Dijk PJ, Bakx-Schotman JT (2004). Formation of unreduced megaspores (diplospory) in apomictic dandelions (Taraxacum officinale, $\mathrm{sl}$ ) is controlled by a sex-specific dominant locus. Genetics 166: 483-492.

Vašut RJ, Vijverberg K, van Dijk PJ, de Jong H (2014). Fluorescent in situ hybridization shows DIPLOSPOROUS located on one of the NOR chromosomes in apomictic dandelions (Taraxacum) in the absence of a large hemizygous chromosomal region. Genome 57: 609-620.

Verduijn MH, van Dijk PJ, van Damme JMM (2004). The role of tetraploids in the sexualasexual cycle in dandelions (Taraxacum). Heredity 93: 390-398.

Vijverberg K, Milanovic-Ivanovic S, Bakx-Schotman T, van Dijk PJ (2010). Genetic finemapping of DIPLOSPOROUS in Taraxacum (dandelion; Asteraceae) indicates a duplicated DIP-gene. BMC Plant Biol 10: 154.

Whitton J, Sears CJ, Baack EJ, Otto SP (2008). The dynamic nature of apomixis in the angiosperms. Int J Plant Sci 169: 169-182. 